\title{
Une mise en lumière des mutations militaires de la fin du Moyen Âge
}

\section{Antoine Leduc}

\section{(2) OpenEdition}

1 Journals

Édition électronique

URL : http://journals.openedition.org/artefact/514

ISSN : 2606-9245

Éditeur :

Association Artefact. Techniques histoire et sciences humaines, Presses universitaires du Midi

Édition imprimée

Date de publication : 1 octobre 2016

Pagination : 307-311

ISBN : 978-2-7535-5174-9

ISSN : 2273-0753

\section{Référence électronique}

Antoine Leduc, "Une mise en lumière des mutations militaires de la fin du Moyen Âge », Artefact [En ligne], 4 | 2016, mis en ligne le 07 juillet 2017, consulté le 14 septembre 2020. URL : http:// journals.openedition.org/artefact/514

\section{(c) (i) $\odot$}

Artefact, Techniques, histoire et sciences humaines est mise à disposition selon les termes de la Licence Creative Commons Attribution - Pas d'Utilisation Commerciale - Pas de Modification 4.0 International. 


\section{Une mise en lumière des mutations militaires de la fin du Moyen Âge}

Antoine LEDUC ${ }^{1}$

Le 25 octobre 1415, la chevalerie française est décimée au cours de la bataille d'Azincourt. Le bilan est terrible et les conséquences désastreuses pour le royaume de France. L'armée française, l'ost féodal composé en grande partie par les vassaux du roi Charles VI, est écrasée par l'infanterie anglaise : les chevaliers sont cloués au sol par une intense pluie de flèches ${ }^{2}$. Les archives conservées ne permettent pas d'avancer des chiffres exacts, mais on estime les pertes à environ 6000 hommes du côté français, contre 600 du côté anglais. Un siècle plus tard, le jeune roi François I ${ }^{\mathrm{er}}$ tient tête à une redoutable armée suisse au sud de Milan et remporte la victoire le 14 septembre 1515 , après deux jours de bataille, la plus longue que la France ait eue à mener jusqu'alors. L'artillerie française y joue un rôle déterminant.

\section{Constat d'échecs et réformes militaires sous Charles VII}

Entre ces deux dates, l'armée française a été transformée en profondeur ${ }^{3}$. En 1415, le constat est sans appel : l'ost, auquel on participe en vertu d'un service féodal, n'est plus adapté pour faire face à des armées plus modernes dans leur organisation et leur mode de combat. Des réformes s'imposent, mais le traumatisme est tel à l'issue de la bataille d'Azincourt et la situation politique intérieure tellement troublée qu'il faut attendre une trentaine d'années avant que Charles VII puisse réorganiser l'armée (illustration 11, cahier couleur). Prélude à cette refonte, une réforme fiscale permet au roi de disposer d'importantes rentrées d'argent. Alors que Charles VII devait auparavant obtenir l'approbation des États généraux de langue d'oc et de langue d'oill pour lever un impôt extraordinaire lui permettant de financer une campagne militaire, il obtient à partir de 1438-1439 que l'impôt 
militaire, la taille, devienne un prélèvement automatique et annuel.

En 1445, la Grande Ordonnance de Louppy-le-Châtel réorganise les troupes. Le roi garde à son service quinze capitaines chargés de recruter chacun une compagnie de cent lances - la lance qui était l'unité tactique de base, comportait six hommes à cheval. Charles VII dispose alors d'une armée de 9000 hommes, soldés en temps de paix et en temps de guerre, au service exclusif du roi et de l'État. Il s'agit de la première armée permanente et professionnelle dans l'histoire de France. C'est avec cette armée, au sein de laquelle les chevaliers laissent place aux " hommes d'armes », que Charles VII met fin à la guerre de Cent Ans, marquée à son terme par deux grandes batailles, celle de Formigny en 1450, qui met fin à l'occupation anglaise en Normandie, et celle de Castillon, le 17 juillet 1453, jour anniversaire du sacre du roi, où les derniers contingents anglais de Guyenne sont taillés en pièces par l'artillerie qui obtient ainsi sa première victoire ${ }^{4}$.

\section{La naissance d'une nouvelle arme : I'artillerie}

Apparue en Occident vers la fin du XIII ${ }^{\mathrm{e}}$ siècle ou le début du XIV ${ }^{\mathrm{e}}$, l'artillerie à poudre était surtout employée pour la défense des châteaux forts. À partir des années 1370, les premières bombardes sont construites à la demande du roi de France et du duc de Bourgogne. Ces bouches à feu de gros calibres sont dès lors utilisées pour l'attaque des forteresses, aux côtés des engins mécaniques (trébuchets, couillards et autres mangonneaux) qu'elles vont progressivement remplacer. L'artillerie à poudre était conçue comme un nouvel outil de démolition et perçue comme telle. Lorsqu'elles étaient réalisées en fer forgé (mais elles pouvaient aussi être coulées en bronze), ces pièces étaient assemblées à la manière des tonneaux : des douves de fer formant la paroi interne du canon sont maintenues par des bagues en fer forgé assurant la cohésion de l'ensemble. Cette subtile technologie repose sur un équilibre entre la pression engendrée par l'explosion (que la pièce absorbe dans une certaine mesure en se déformant) et le coefficient de résistance des matériaux. Des études récentes ont montré que le secret de fabrication de ces pièces est en partie lié à la pression de serrage qu'exercent les bagues sur les douves. À partir des années 1430 , ce mode de fabrication est parfaitement maîtrisé : des pièces monumentales sont produites, à l'image de la célèbre Dulle Griet, qui existe encore sur la place du marché de la ville de Gand : elle mesure plus de 8 mètres de long et pèse plus de 16 tonnes.

À la même période, on découvrait avec horreur les dégâts causés par de petites armes à feu portatives, les couleuvrines. Ancêtres de nos fusils, les couleuvrines tiraient de petites balles de plomb dont les chroniques rapportent que «qui en étoit frappé à peine povoit-il eschapper sans mort ». Pour créer une artillerie de campagne, il fallait donc augmenter la taille de ces armes portatives pour les 
rendre efficaces à l'échelle d'un champ de bataille. Mais les bouches à feu en fer forgé ne résistaient pas à la pression exercée par un boulet métallique deux à trois fois plus dense qu'un boulet de pierre. Il fallait donc modifier à nouveau le procédé du montage en tonoille pour les pièces en fer ou, mieux, utiliser des canons en bronze. Entre 1450 et 1460, les serpentines constituent la première artillerie de campagne (illustration 12, cahier couleur) : il s'agit de pièces légères, montées sur des affûts à roues spécialement conçus pour leur transport, et tirant des boulets de calibre moyen, en fer ou en plomb. L'artillerie de campagne (tube en bronze à chargement par la bouche, poudre noire et boulet métallique) ne connaît plus d'innovation technique avant le milieu du XIX $x^{\mathrm{e}}$ siècle.

Cette armée nouvellement organisée et équipée d'une redoutable artillerie permet de mettre un terme à la guerre de Cent Ans et assure les premières victoires françaises au début des guerres d'Italie ${ }^{5}$.

\section{Un rassemblement de pièces majeures}

La plupart des pièces d'armement réunies dans l'exposition provenait des collections anciennes du musée de l'Armée, des Royal Armouries et du Mary Rose Trust. L'exposition a bénéficié de prêts prestigieux, fruits d'une collaboration enrichissante avec plusieurs institutions françaises et étrangères ${ }^{6}$.

La Bibliothèque nationale de France s'est montrée particulièrement généreuse. Une dizaine de manuscrits enluminés et incunables sont sortis spécialement des réserves : chroniques illustrant les épisodes de la guerre de Cent Ans, traités d'ingénieurs militaires italiens ou encore partition des chansons glorifiant la victoire de Marignan. On pouvait aussi admirer les Statuts de l'ordre de saint Michel, exemplaire personnel du roi Charles VIII, seul manuscrit connu enluminé par Jean Hey.

Pour célébrer la fin de la guerre de Cent Ans et sa victoire sur les Anglais, le roi Charles VII a fait frapper jusqu'à la fin de son règne des médailles commémora- tives qu'il offrait en étrennes. Trois exemplaires de ces Calaisiennes, en or, étaient prêtés par le département des Monnaies, Médailles et Antiques.

Parmi les pièces majeures de cette exposition se trouvait également le chapel doré de Charles VI, conservé au musée du Louvre. Réalisé vers 1400, il s'agit de la plus ancienne pièce d'armure royale française conservée de nos jours. Plusieurs pièces d'artillerie issues des collections suisses ou de celle du musée de l'Armée permettaient d'évoquer la naissance de cette arme nouvelle ainsi que les grandes phases de mutation qui l'ont affectée au cours du $x^{e}$ siècle. La plus impressionnante d'entre elles était sans nul doute la bombarde prêtée par le musée historique de Bâle : elle pèse plus de deux tonnes.

Parmi les œuvres d'art, la présence tout à fait exceptionnelle de l'ange en bronze, venu tout spécialement de la collection Frick à New York, révélait l'étendue du savoir-faire des canonniers 
royaux (illustration 13, cahier couleur). Daté de 1475, il a été coulé par Jean Barbet, un canonnier de Louis XI, originaire de Lyon. Il s'agit en outre de la plus grande sculpture française en bronze conservée pour cette période. Les canon- niers royaux étaient des fonctionnaires chargés de la fabrication des pièces d'artillerie pour l'armée, mais ils pouvaient aussi répondre à des commandes de mobilier civil ou religieux.

\section{Un nouveau regard sur d'anciennes collections}

Cette manifestation a été l'occasion de découvrir certaines œuvres méconnues du public, comme cet orgue à douze canons (illustration 14, cahier couleur). Ancêtre dela mitrailleuse, cette pièce d'artillerie datant de la fin $d u x^{e}$ siècle permettait de tirer une salve de douze balles de plomb. Il semble que ce soit le seul orgue médiéval conservé aujourd'hui. Il a été redécouvert à la faveur d'un travail d'inventaire de la collection de bouches à feu du Moyen Âge du musée de l'Armée. Pour l'exposition, un affût a été spécialement reconstitué d'après une enluminure du début du $\mathrm{xvI}^{\mathrm{e}}$ siècle : il permettait aux visiteurs de visualiser la pièce dans son ensemble.

Le rassemblement des œuvres présentées dans l'exposition a également permis un travail de comparaison. La Relation du voyage de Gènes, rédigée par Jean Marot, relate l'action répressive de Louis XII à l'encontre de la révolte de Gênes en 1507. Les enluminures, réalisées par Jean Bourdichon en 1507-1508, représentent un armement conforme à celui utilisé par les troupes françaises au début $\mathrm{du} \mathrm{XvI}^{\mathrm{e}}$ siècle. Les hommes d'armes sont revêtus de harnois milanais bien identifiables. Au premier plan d'une autre composition, deux canons en bronze sont inspectés par des canon- niers; leur décor est identique en tout point à celui d'une pièce d'artillerie française coulée à Lyon en 1507 et conservée au musée de l'Armée. La confrontation des pièces conservées et de leur représentation montre que le travail de l'enlumineur se veut résolument figuratif et réaliste.

Parmi les pièces révélées par l'exposition, une armure de cavalier germanique tenait une place de choix (illustration 15, cahier couleur). Prise à l'armurerie de Neuburg par les troupes françaises en 1800, elle a ensuite été envoyée au musée des Beaux-Arts de Rennes où elle était conservée en réserve depuis 1802. Réalisée vers 1535 par l'armurier Valentin Siebenbürger, elle était destinée à un proche de l'électeur palatin Otto Henri. Remarquable par le soin apporté à sa réalisation (bordures gravées à l'eau-forte et dorées), cette armure était présentée sur un cheval équipé d'une barde provenant du même arsenal. L'ensemble, présenté sur le côté d'un carré de piquiers suisses, permettait d'évoquer la gendarmerie cuirassée du début $\mathrm{du} \mathrm{xvI}^{\mathrm{e}}$ siècle. L'armure, mise en dépôt au musée de l'Armée, a intégré les salles d'exposition permanente à la fin de cette manifestation. 


\section{Notes}

1. Doctorant en histoire médiévale sous la direction de Bertrand Schnerb (université de Lille 3). Assistant de conservation puis adjoint du conservateur au département artillerie du musée de l'Armée (2007-2015). Ses axes de recherches concernent l'artillerie ancienne, l'histoire des collections d'armes, les faux et pastiches dans les collections d'armes anciennes. Contact [antoineleduc@wanadoo.fr].

2. Valérie Toureille, Le drame d'Azincourt. Histoire d'une étrange défaite, Paris, Albin Michel, 2015.

3. Philippe Contamine, Guerre, État et société à la fin du Moyen Âge. Études sur les armées des rois de France. 1337-1494, Paris, Mouton, 1972.

4. Nicolas Le Roux, Le crépuscule de la chevalerie. Noblesse et guerre au siècle de la Renaissance, Seyssel, Champ Vallon, 2015.

5. Cédric Michon (dir.), Les conseillers de François I Ir, Rennes, PUR, 2011.

6. Antoine Leduc, Sylvie Leluc, Olivier Renaudeau (dir.), D'Azincourt à Marignan, Chevaliers \& bombardes, 1415-1515, Paris, Gallimard, Musée de l'Armée, 2015. 